\title{
Pour une rhétorique du quotidien dans la littérature québécoise moderne*
}

Danielle Forget**

RÉSUMÉ : Que ce soit sous la forme quasi-orale des monologues de Clémence DesRochers, ou dans la trame narrative du roman de Michel Tremblay, Des Nouvelles d'Édouard, une rhétorique du quotidien se profile dans les œuvres littéraires québécoises; elle prend pour toile de fond la réalité individuelle quotidienne et semble activer le familier afin de provoquer chez le lecteur une distance critique. Par des figures à portée textuelle comme le cliché et l'ironie, elle offre alors à la réflexion une transgression des normes littéraires et suggère un dépassement de nos réflexes d'interprétation.

\section{Introduction}

Notre propos ne s'attachera pas à ces œuvres littéraires où la grandiloquence s'affiche, où le débat s'élève vers l'essence de l'homme ou encore vers les grandes questions socio-historiques. Nous privilégierons plutôt des œuvres accusant une tendance qui, tout en étant difficile de la circonscrire en courant littéraire, laisse néanmoins

- Recebido para publicaça em julho de 2000.

“Professeur au Département de Léttres françaises de l'Université d'Ottawa. 
des marques dans les préoccupations littéraires québécoises et que nous désignerons comme la rhétorique du quotidien.

Comment la caractériser ? Elle suggère, bien sûr, une préférence pour un contenu alimenté au train-train de tous les jours, mais elle ne se limite pas à ces questions de contenu. Nous la retrouvons plutôt dans ces œuvres qui cherchent à dédramatiser des questions fondamentales en les abordant du point de vue de l'individu aux prises avec la réalité de tous les jours dans une intention manifeste de faire ressortir le contraste entre le concret et l'abstrait.

Nous tenterons de voir comment cette rhétorique du quotidien se manifeste dans certains textes littéraires québécois, plus particulièrement dans quelques monologues de Clémence DesRochers, et dans un roman de Michel Tremblay, Des Nouvelles d'Édouard. Elle se trouve favorisée, comme nous le verrons, par l'ironie et le cliché, deux figures qu'affectionnent particulièrement ces deux auteurs québécois et qui ont contribué à leur renommée.

\section{Clémence DesRochers : de la parole mise en scène au récit du quotidien}

Quand on habite le Québec, il est impossible de ne pas connaître Clémence DesRochers, "Clémence" comme on l'appelle. Elle est tout à la fois poète, chanteuse, comédienne, organisatrice de revues et surtout monologuiste. C'est, en effet, par ses monologues qu'elle combine le mieux ses talents pour l'écriture et la scène, comme "poétesse du geste et de la parole", pour employer sa façon de se présenter.

Elle a opté pour des monologues percutants sur la réalité sociale et, plus particulièrement, sur la place de la femme dans cette réalité. Son ton est toujours moqueur, parfois tendre et souvent amer. Il n'est donc pas étonnant de retrouver des formes comme l'ironie, le cliché, mis au service de son propos, qui nous séduit d'abord par l'humour. 
Mais l'humour est une notion bien vague qui englobe toutes sortes de manifestations ayant en commun, finalement, l'effet qu'elles produisent, à savoir le rire à des degrés divers. Y. Allard remarque fort justement:

C'est qu'il y a plusieurs nuances d'humour, mais qui toutes, au rebours de l'ironie, maîtresse morale et réformatrice, tendent plutôt à la tolérance exorcisant les angoisses sociales, dédramatisant les situations inévitables, désamorçant l'agressivité naturelle et surtout, par complicité, connivence et même parfois pitié, retrouvant par innocence, jusque dans la contradiction, les constantes réalistes de l'humain faible, fragile et tellement semblable à soi-même. (ALLARD, 1979:561)

L'humour, chez Clémence DesRochers procède essentiellement de cette connivence avec le spectateur qu'elle construit systématiquement malgré l'apparente improvisation et désinvolture des propos. On ne peut comprendre le monologue sans faire référence au thêâtre dont il est une forme particulière. Même si le monologue prend souvent l'allure d'un récit, il est avant tout un discours tenu sur scène devant un spectateur et développant une question sur le mode tragique ou humoristique. La parole mise en scène en constitue la dimension essentielle même si, comme l'a fait remarquer Larthomas (1972) pour le langage dramatique en général, elle a fait préalablement l'objet d'une élaboration par écrit (ce qui empêche de la voir comme un discours spontané) et qu'elle recevra vraisemblablement une diffusion écrite. L'analyse rhétorique des monologues de Clémence DesRochers doit donc prendre en considération le récit et le cadre discursif dans lequel il s'insère. ${ }^{1}$

Attachons-nous au cadre discursif. Il est important de tenir compte d'un premier niveau qui renvoie directement à la situation d'énonciation s'instaurant entre le monologuiste-locuteur et son

\footnotetext{
'Nous adopterons la position bien connue, reformulée par J. Adams, et qui consiste à associer acte énonciatif et fiction: "One such feature is that a fiction must have a communicative context as part of its linguistic structure, for in order to present a lietional world with language, speceh acts must be performed, and they must be performed by someone and in some contexl" (ADAMS:23)
} 
auditoire, le plus souvent dans les premières minutes de l'entrée en scène. ${ }^{2}$ Même si ce moment semble à première vue se constituer comme "l'extérieur du texte" ou "l'avant-monologue", cette dimension est essentielle car elle justifie la prise de parole du locuteur, elle s'ajuste aux réactions en cours de spectacle; autrement dit, elle concerne la production et la réception du discours et prend place dans un cadre chronologique, qui, nous le verrons, sert de bornes aux autres niveaux. Et c'est par là que le monologue instaure un mode particulier de communication avec son auditoire à partir du familier.

Une mutation s'effectue souvent dans les premières minutes du monologue. Du collectif on passe au particulier: le monologuiste s'adresse non plus à son public mais il devient lui-même narrateur ou personnage et la conversation se privatise. Quant au public, il reçoit lui-même un rôle: il devient l'oreille attentive devant le narrateur qui raconte ou le témoin d'une action se déroulant entre personnages.

Les niveaux, appelons-les discursifs et narratifs, se superposent et ce sont les personnages qui entrent en scène directement sans être introduits par l'intermédiaire d'un narrateur: par exemple, lorsque le monologue prend la forme d'une conversation téléphonique, comme dans "Valentine, Saint-François et la publicité" (DESROCHERS, 1986:69-70) l'amorce et la fin de celle-ci servent aussi d'entrée en matière et de clôture du monologue. Il va sans dire que ce genre favorise l'insertion du quotidien, mais son efficacité rhétorique tient à d'autres raffinements, comme nous le verrons. Dès les premiers moments du monologue "Valentine, Saint-François et la publicité", nous comprenons que la conversation téléphonique n'a pas un but

\footnotetext{
2 "Le monologue est peut-être, aujourd'hui comme au temps de Jean Narrache et de Fridolin, notre forme de théâtre la plus vraie, la plus populaire et la mieux engagée, la seule où puissent se retrouver ensemble travailleurs et chômeurs, intellectucls el bourgeois." (MAILHOT, 1973 : 26) Dans ce récit, se trouvent des personnages à qui on donne directement la parole. Les personnages sont autonomes: ils parlent, ils agissent et nous sommes ténoins de ce qui leur arrive.
} 
spécifique mais exprime le flottement de celui qui a un simple désir de parler, comme l'atteste le "Qu'est-ce que tu fais?".

Allô Odette ! C'est Valentine. Qu'est-ce que tu fais ? Quel film ? J'aime pas les films de cow-boy. As-tu déjà vu le film 'Les chemins de la lumière'? Ça c'est beau. C'est un chef-d'œuvre. Un chef d'œuvre, Odette, c'est quelque chose qui dure très longtemps. Ça commencé à sept heures, ça fini à onze heures.

C'est l'histoire de Saint-François d'Assise, un moine. Le connais-tu ? Odette, il pouvait pas faire l'annonce de Corn flakes, il vivait au treizième siècle, le Kellogg était pas inventé. (...) (DESROCHERS, 1986:69-70)

Livrée à la spontanéité des interventions, la conversation s'apprécie par le développement erratique des thèmes et par les rapports entre les personnages qui se dessinent. Odette, qui a des goûts en matière de cinéma que l'on qualifie souvent de simples les films de cow-boy -, atteste une ignorance certaine, ne sachant pas ce qu'est un chef-d'œuvre. Le début de la conversation téléphonique est à reporter sur le plan discursif: la mise en place de "I'univers familier" se fait dès les premiers moments. Tout au long du monologue, le personnage d'Odette continuera de décevoir Valentine obligeant cette dernière à effectuer des rectifications dans ses propos, à lui expliquer des termes qu'elle ne comprend pas, etc.

La distinction de ces niveaux intervient directement dans les mécanismes mis en oeuvre par les figures, ce qui permet le déclenchement du rire. La situation observée et jugée est le plus souvent celle qui se déroule entre les personnages: Valentine et Odette au téléphone, l'épouse qui raconte son voyage. Comme l'ironie et les autres figures supposent une distance, un regard critique, ce n'est habituellement pas au niveau des personnages qu'elle se produit, $\mathrm{du}$ moins dans les monologues de Clémence DesRochers.

C'est précisément lorsque le monologue bascule graduellement dans la fiction que les niveaux de situation changent et que s'amorce graduellement l'insertion du récit. Le narrateur a alors le souci de raconter fidèlement des événements dont il a été témoin ou dans 
lesquels il a été impliqué. Le narrateur introduit les personnages et en ce sens, il est l'intermédiaire fictif entre une situation mettant en scène des personnages que le discours construit et la situation d'énonciation réelle qui réunit le monologuiste et son public. Mais il le fait le plus souvent en misant sur la vraisemblance, à travers une fidélité prétendue aux événements. Le récit est alors amorcé par des relais: 'j'ai rencontré l'autre jour..", ou "un exemple..". En tant que spectateurs ou lecteurs, nous devenons ainsi confidents.

Ces différents niveaux de structuration du monologue se révèlent de façon plus ou moins accentuée selon les textes, un niveau d'intervention en cachant un autre le plus souvent. Il semble toutefois y avoir une constante: la fonction du monologue qui consiste à faire rire le spectateur est le plus souvent occultée et n'intervient jamais explicitement. Le monologuiste se montre comme narrateur improvisé qui pense tout à coup à quelque chose, qui doit livrer le fruit de sa réflexion, raconter une conversation étonnante, etc, autant de manières de partager avec le spectateur-lecteur un présent spatio-temporel, à partir de repères comme le je-ici-maintenant; le genre lui-même ouvre donc une porte sur le quotidien.

\subsection{L'univers des personnages et le cliché.}

Dans ses monologues, Clémence DesRochers met en scène des personnages qui évoluent dans un univers reconnaissable: le milieu est québécois par les noms des vedettes auxquelles il est fait référence, les publicités qui y sont citées, le renvoi au bien-être social (le B.S, qui est l'assistance sociale). Des situations-types sont invoquées: le père qui travaille pour l'éducation de ses enfants, le grand frère qu'on cite comme modèle, etc.

Les particularités linguistiques participent du décor. En effet, la langue donne dans le registre familier ou accuse des traits populaires évidents: distorsion dans la prononciation ("la drogne" pour "la 
drogue"); licence sur le plan des genres ("une appartement"); problèmes de construction ("Laquelle annonce de bière aimes-tu la mieux?"). Ailleurs, comme dans le monologue, "On a fait un ben beau voyage franch'ment hein Armand? " (DESROCHERS, 1973:2324), la langue est tout simplement expressive par la mise en oeuvre de moyens souvent typiques de l'oral et aisément reconnaissable. Par exemple, l'épouse, au retour de son voyage organisé, y va d'un accent d'insistance en s'attardant sur le " $r$ " de "très" ("Un trrrrrès beau voyage").

Du point de vue théorique, il est important de comprendre que ces images du monde quotidien s'organisent en "scripts", c'est-àdire, en sortes de scénarios où événements, décors et comportements s'intègrent au savoir partagé culturel. Il suffit d'un seul élément pour déclencher chez le destinataire une série cognitive d'images-types: il ne s'agit pas d'une vision statique mais dynamique qui laisse prévoir les réactions, les répliques possibles par référence à des schémas collectifs. De là viennent les "attentes" respectées ou contredites dont se nourrit par la suite le récit.

S'il est vrai que la plupart des monologues de Clémence DesRochers s'organisent autour d'un thème central et qu'une signification d'ensemble détermine la visée du texte, ce processus passe obligatoirement par un renvoi à la réalité sociale, politique. Ainsi, le renvoi au contexte social ne sert pas uniquement de "toile de fond" à la narration: il sera constamment travaillé. Le contexte, même s'il apparaît familier pour le spectateur dès les premiers moments du monologue, est appelé à se transformer ou du moins à recevoir un éclairage particulier en cours d'élaboration, ce qui lui confère un aspect dynamique.

Le recours au cliché, comme forme verbale usée et reconnaissable, tout en étant à la source de la dimension caricaturale des personnages et garant de l'humour, est loin de ressasser la banalité; il s'appuie néanmoins sur nos modes de raisonnements habituels, sur ce qui compose notre vie quotidienne jusque dans sa réalité la plus absurde 
ou cocasse pour procéder à des remises en question. En effet, en tant qu'emploi verbal figé, le cliché apparaît comme une opinion toute faite mais qui se double de l'utilisation d'une séquence verbale aisément repérable parce qu'elle s'insère dans la mémoire collective comme répétition. Ces deux caractéristiques essentielles, suggérées par les travaux d'Amossy et Herschberg Pierrot (1997), nous permettent de le distinguer du stéréotype. Ainsi le cliché signale non seulement une appartenance à d'autres discours mais le caractère répété d'une séquence. Il mise donc sur cette propriété pragmatique de la reconnaissance qui oriente le lecteur vers l'interprétation souhaitée tout en provoquant son questionnement.

Le monologue "Valentine, Saint-François et la publicité" (DESROCHERS, 1986:69-70) recèle plusieurs clichés. D'abord, lorsque Odette et Valentine discutent de leurs préférences en matière de cinéma, Valentine vante un film sur Saint-François d'Assise, Les chemins de la lumière en ayant recours à l'argument du "beau" par une formule catégorique qui tient du cliché: "Ça c'est beau. C'est un chef-d'œuvre". Dans l'histoire du film telle que nous la raconte le personnage, Saint-François se rebelle contre les "conditions de travail", ce qui entraîne Odette à tirer des conclusions qui accusent un réflexe social: la facilité du recours à l'Etat en cas de perte d'emploi, mais avec une formulation qui trahit le milieu québécois (le "B.S.", c'est le Bien-être social, un organisme gouvernemental d'assistance sociale) et les préjugés s'y rapportant:

Facile à dire: y avait qu'à se mettre sur le B.S. Le bien-être social n'existait pas au Moyen Age, pauvre Odette. Le saint homme pouvait pas partir à l'aventure avec son chéque d'une main et sa caisse de bière de l'autre. (DESROCHERS, 1986:69-70)

Amené par inférence, le cliché reprend une vision négative des assistés sociaux véhiculée dans la société québécoise: "ceux qui bénéficient du Bien-être social vivent bien et boivent bien!". La figure relève d'une critique sociale: on feint de présenter ces situations ou comportements comme la "normalité" alors que le ton ironique se 
profile, comme un hors-texte cependant. En effet, les personnages portent rarement de regards critiques sur eux-mêmes et le monde dans lequel ils vivent: la critique et le rire proviennent de la connivence entre le monologuiste-narrateur et les spectateurs.

Le renvoi au quotidien aide à construire cette connivence. Valentine poursuit le récit de sa soirée face à l'écran de télévision avec ses parents. Sa mère se lève, lorsque le film est interrompu par les messages publicitaires. "..vous me direz qu'est-ce que j'ai manqué", sous-entendant "..si le film recommence avant que je ne revienne". Ce cliché sort tout droit de nos habitudes quotidiennes et fait sourire parce qu'il nous est montré comme appartenant au domaine collectif: c'est tout à la fois la formulation et la situation stéréotypée que l'on ne peut manquer de reconnaître.

Un peu à la manière de la citation, le cliché se désigne lui-même comme un corps étranger. Issu de la banalité, de la naïveté, il se produit dans un mouvement de conformité avec l'extérieur du texte- répétition du dire collectif, ce qui lui assure en même temps une certaine vraisemblance au niveau du texte. Les clichés sont amplifiés par un développement textuel qui montre l'insistance sur le sujet. Lorsque Valentine valorise le film par l'argument du chef-d'œuvre, elle sera forcée par Odette de le définir et elle le fera d'une manière qui atteste sa maladresse avec les idées reçues. Si l'on est tenté de traduire "qui dure très longtemps" par "qui se transmet à la postérité", l'énoncé qui suit nous en détourne en ramenant le sujet sur un terrain trivial, celui de la durée du film. L'humour surgit souvent dans ces moments où les attentes sont trompées, comme l'a démontré fort justement Violette Morin (1966) à propos des disjonctions dans les histoires drôles qui ont justement pour effet de faire dévier le discours dans une direction inattendue.

Souvent le cliché est prolongé par un développement qui se montre en conformité argumentative avec lui. Lorsque la mère demande qu'on lui raconte ce qu'elle va perdre du film par son absence, l'énoncé qui suit reprend les présupposés mais en donnant la liste 
des commerciaux qu'elle a ratés: "Elle a manqué Stay free, Wonder Bras, Head and Shoulders, et la p'tite Simard qui court dans les champs avec ses poudings Laura Secord." A part la note humoristique que comporte le recours à tous ces termes anglais - les compagnies sont souvent américaines et les annonces qui sont présentées sur les chaînes francophones proviennent souvent de traductions de celles présentées sur les chaînes anglophones - c'est la réponse qui produit la surprise et trompe les attentes du lecteur, en orientant la conversation vers le trivial tout en s'interdisant d'approfondir le sujet du film pour rester en surface des conditions de son visionnement.

Lorsque ce décrochage intervient, la distance critique qui l'accompagne ne peut être rapportée au personnage - car cela viendrait en contradiction avec son comportement et des propos favorables qu'il tient sur la publicité - mais au narrateur et c'est uniquement par ce biais que se justifie la perception de l'ironie.

\subsection{Les enchaînements ironiques}

Les monologues de Clémence DesRochers sont avares de commentaires pouvant effectuer un retour distancié sur la situation des personnages. Par exemple, dans le monologue que nous avons étudié, aucun jugement direct n'apparaît sur le comportement de Valentine; si cette dernière en porte sur Odette, il s'agit de réactions intégrées aux dialogues qui ne relèvent par de l'ordre métadiscursif.

C'est plutôt à l'ironie que reviendra la fonction critique des commentaires. On peut se demander la raison d'une telle exploitation de la figure. Il semble que le déclenchement de la distance critique s'en trouve facilité: plutôt que d'amener la réflexion directement sur une question d'actualité (ou du moins appartenant à l'univers de référence comme la publicité) et risquer une formulation plus compromettante du type: "De nos jours, la publicité occupe une place envahissante" ou "Il faut réduire le temps d'antenne réservé aux annonces publicitaires", la démonstration procède de l'implicite en 
exploitant les figures, ainsi que les inférences textuelles qui leur sont associées. La distanciation nécessaire à la perception de l'ironie condition de son interprétation comme détournement du sens s'appuie de façon caractéristique, dans ce monologue, sur les particularités réductionnistes et aliénantes du quotidien.

Examinons quelques-uns de ces enchaînements qui sous-tendent l'argumentation tenue par les personnages: "François a un rêve: rebâtir l'église de la paroisse en ruine, et fonder une nouvelle religion." Elaboré sur une référence biblique, on attend de la suite de cet énoncé une précision quant à cette "nouvelle religion". Mais l'enchaînement est désarmant de trivialité: "Il réunit ses meilleurs amis, leur tond les cheveux et les habille tous en brun foncé". Il y a donc réduction.

Notons aussi un type d'anachronisme qui vient de la confusion du personnage entre la réalité de son univers de référence avec celui du récit enchâssé, lorsque plus loin Valentine mentionne:

La nouvelle religion de François dit au monde que posséder des affaires ne rend pas heureux. Acheter, acheter tout ce qu'on annonce, c'est pas ça le bonheur.

l'emploi de "annonce" est discordant dans ce qu'on a tout lieu de prendre pour un discours rapporté indirect. Sur le plan textuel, toutefois, cela opère un rapprochement avec la question de la publicité. Ou encore, que dire de la suite: "Le bonheur, je ne sais pas ce que c'est. J'ai pas entendu."

Sous des apparences anodines, l'argumentation du personnage se développe par failles, à coup de répliques désarmantes qui minimisent l'importance du sujet et le subordonne au trivial. Le personnage de Valentine apparaît dans toute sa naïveté, d'autant plus que l'équivoque liée au segment "je ne sais pas ce que c'est" semble faire dire par le personnage le commentaire qui pourrait être celui, ironisant, du narrateur. Il semble ainsi que l'histoire est un prétexte pour mieux faire apparaître une dimension argumentative: en amenant l'auditeur à une distance critique, elle marque le sens figuré. 
Dans quelques monologues de Clémence DesRochers, et plus particulièrement dans celui étudié, un itinéraire énonciatif vient s'ajouter et jouer un rôle similaire à l'itinéraire narratif. Il règle certains enchainements par le biais cette fois des interactions verbales entre Valentine et Odette. Subordonné au récit du film, il justifie des arrêts, des retours en arrière ou des ajouts d'information. Nous n'avons pas accès aux interventions du personnage d'Odette mais nous les devinons par ce que nous en montrent les répliques de Valentine. Les enchaînements qui relèvent de la communication entre les personnages sont plus lâches que ceux suivant un cours narratif. La progression, il faut dire, se fait au gré des interruptions imaginaires d'Odette; si quelques répliques sont sollicitées par Valentine, la plupart sont imprévisibles et commandées par un manque de compréhension des éléments du récit. Ce sont les plus marquantes, celles où Valentine doit lui préciser ce qu'est un chef-d'oeuvre, ce que veut dire "sexiste", etc. Tout en respectant, l'à-propos du texte, ces interventions donnent lieu à des effets comiques savoureux. Elles construisent l'image des personnages.

En portant notre attention à l'organisation d'ensemble du monologue, nous remarquons l'intervention d'un parallélisme qui lie l'itinéraire narratif (récit du film) et l'itinéraire communicationnel (interaction des personnages): il procède de la sélection des éléments de l'histoire de façon à reproduire, dans le cours du monologue, l'alternance entre le temps du film et les moments consacrés aux annonces publicitaires. En effet, une telle alternance, qui relève du mode iconique, accapare le déroulement textuel et fait que les remarques sur les publicités sont thématiquement plus envahissantes que le récit du film lui-même. L'aliénation des personnages par la publicité trouve une preuve dans leur comportement qui consiste à fusionner ainsi des éléments appartenant à deux mondes référentiels distincts (le réel et la propagande): on en arrive au paroxysme quand les personnages vont même jusqu'à se servir des annonces publicitaires pour identifier les personnages du film. Ainsi l'attitude ironique 
ressort le mieux à ce niveau supra-narratif qui ne peut être saisi qu'en misant sur la capacité de l'auditeur à changer de niveau d'interprétation: entraîné par les différents décrochages suscités par les figures, il est en alerte et vraisemblablement apte à apprécier cette subtilité sur le plan de l'organisation textuelle. Le trivial tel qu'il apparaît dans le déroulement du quotidien anime le texte en courtcircuitant toute tentative d'élaborer une réflexion approfondie et autonome; il s'interpose comme marque d'aliénation. Citons un dernier passage, emprunté cette fois à "On a fait un ben beau voyage franch'ment hein Armand?" (DESROCHERS, 1973:23-24):

L'Italie. C'est de toute beauté l'Italie, hein Armand? Nous avons visité les villes principales comme Venise... ensuite nous avons vu Venise et puis...c'est très beau Venise... Mais c'est pas très propre, avec toute cette eau dans les rues, vous comprenez que c'est difficile d'entretien.

Le caractère inhabituel de ce voyage pour les protagonistes apparaît, à travers le ton ironique du narrateur, par une répétition suspecte qui camouffle en fait l'oubli, pour ne pas dire l'ignorance d'autres noms de villes, et aussi par les préjugés de malpropreté importés des valeurs locales. L'enracinement dans le quotidien transparait, non seulement dans ces procédés, mais aussi dans le calque des conversations typiques relevant de ce genre de situations, un cliché conversationnel en quelque sorte.

\section{Michel Tremblay et les jeux avec le quotidien}

En s'adjoignant des figures comme le cliché et l'ironie, la rhétorique du quotidien colore et infléchit la trame narrative en lui conférant une certaine orientation. Tout en différant d'un écrit à un autre, cette orientation s'appuie sur les propriétés pragmatiques qui expliquent son fonctionnement. Si les monologues de Clémence DesRochers s'ingéniaient à démontrer une conformité aux lois du 
quotidien (et parfois sa suprématie), le roman de Tremblay, Des Nouvelles d'Édouard (1984) semble miser sur le contraste. ${ }^{3}$

Le roman construit l'histoire d'Édouard qui vient de gagner en héritage un voyage en France par transatlantique en première classe. La destination Montréal-Paris n'était pas en 1947, année où se situent les événements, aussi fréquentée qu'elle l'est maintenant, ni la culture française aussi connue pour un Québécois. Aux yeux d'Édouard, modeste vendeur de chaussures dans un quartier chic de la ville, ce voyage constitue un rêve, celui de connaître les mœurs et la culture auxquelles il n'a accès qu'à travers les films diffusés. Le personnage mène aussi une vie fantaisiste à ses heures puisqu'il sévit comme travesti, en tant que Duchesse de Langeais, dans les milieux populaires des cabarets, ceux bien connus de la rue Saint-Laurent à Montréal. Ce double aspect de sa personnalité sera constamment présent dans le récit du voyage qu'il entreprend sous prétexte de tenir un journal.

Des Nouvelles d'Édouard construit un cadre discursif particulier reposant donc sur le journal sans toutefois que des dates en segmentent la relation des événements; il prend plutôt l'allure d'une conversation et le narrateur s'implique par une voix autobiographique qui s'adresse à sa belle-sœur, en tant que destinataire marquée dans le texte. Mais, par ricochets, c'est nous, lecteurs, qui sommes visés. Comme cette belle-sœur fait partie de son entourage familier (lorsque Édouard ne voyage pas), il s'agit là à la fois d'une justification et d'une caution du quotidien. En effet, c'est afin de lui faire connaître les moindres détails de son voyage, comme si elle voyageait à ses côtés, qu'Édouard se propose de tenir une relation précise des événements. De plus, il pourra se permettre d'être naturel dans le ton employé pour rédiger ce journal en toute intimité, par contraste avec les prétentions de comportement qu'il se croira forcé d'adopter en société alors qu'il

'Des Nouvelles d'Édouard est le quatriène tome des Chroniques du P'lateau Mont-Royal, metlant en scène Édouard en tant que Duchesse de Langeais, après La grosse femme d'à côté est enceinte, Thérèse et Pierrette à l'école des Saints-Anges, La duchesse et le roturier. 
est confronté à une classe sociale qui n'est pas la sienne. En conséquence, Édouard assume deux rôles énonciatifs: celui de narrateur d'événements mais aussi celui d'interlocuteur pour sa bellesœur, d'où l'alternance entre un mode narratif et un autre, discursif. Dans le mode narratif, le journal prend l'allure d'un récit, laissant alors tomber toutes marques autoréférentielles d'une écriture en élaboration et se concentrant sur la trame des événements allant jusqu'à donner la parole à des personnages. Dans le mode discursif, le cadre du journal apparaît, prenant des allures quasiconversationnelles comme nous l'avons mentionné plus haut; le personnage s'y révèle sous un jour plus intime.

En dépit du fait que cette croisière en transatlantique constitue un événement unique dans la vie d'Édouard, l'univers du personnage se compose au quotidien dans le roman. Bien sûr, le journal en est la cause principale mais aussi le rythme des événements à bord marqués par un temps habituel (rencontres et emplois du temps répartis le matin, le midi et le soir). Il est important de souligner l'emploi particulier du présent. Il actualise ces événements et estompe ainsi la réalité d'une écriture postérieure - forcément - aux événements vécus, de même que celle d'une lecture différée de ce journal par rapport au moment de l'écriture; bref, le temps de l'histoire, de l'écriture et de la lecture semblent coïncider, ce qui contribue à nous faire vivre (nous, lecteurs) ce quotidien.

Au cours de la traversée, propulsé dans un milieu social qui n'est pas le sien, Édouard tentera d'adopter les bonnes manières de la haute société, non sans difficultés, ou bien alors il affichera par dépit sa différence sociale en l'exagérant. Dans l'un et l'autre cas, il en ressort un contraste social et un malaise, celui du personnage, qu'il communique au lecteur par phénomène d'identification, alors qu'à d'autres moments, c'est le lecteur qui se distancie de ce personnage humilié, diminué. Il se plaît, surtout au début de son voyage, à se composer un rôle à la hauteur de la situation mais qui contraste avec ce qu'on estime être sa réalité quotidienne: 
Antoinette Beaugrand s'est arrêtée à ma hauteur, les yeux rivés sur mon cahier.

- Excusez-moi de vous poser une question aussi indiscrète, mais cela me brûle les lèvres depuis l'autre jour...

- Allez-y, madame, il faut toujours calmer ses feux...

(Peut-être un peu raide, comme début, mais c'est sorti tout seul.)

- Vous écrivez votre journal?

Je ne sais pas pourquoi ni comment mais j'ai senti comme une sorte de mépris au cœur de sa curiosité, comme si écrire son journal en public comme je le faisais, avait quelque chose de déplacé et même de vulgaire. Elle doit penser que Julien Green se cache pour écrire le sien, même s'il le publie.

- Non, j'écris un essai...

(...)

Là, évidemment, je me suis rendu compte de ma gaffe. J'avais encore parlé trop vite. Je connais à peine le mot essai, je ne suis pas sûr de savoir exactement ce que ça veut dire, imaginez-vous si j'ai patiné pour me construire une réponse! J'ai pensé vite, j'ai parlé vite et j'ai vite compris que je venais de commettre ma seconde erreur :

- J'essaie de prouver l'influence du théâtre National sur la vie artistique de Montréal...

Le sourire jusque-là engageant d'Antoinette Beaugrand s'est figé sur son visage comme une framboise écrasée.. (TREMBLAY, 1984:106-107)

Malgré le rôle qu'il se compose, Édouard dévoile ses origines en faisant allusion à un théâtre associé aux goûts populaires de l'est montréalais, secteur non huppé et donc, ne recevant pas les faveurs des milieux aisés auxquels se targue d'appartenir son interlocutrice. Dans les commentaires qui suivront cet état de fait, le personnage libérera sa frustration; il ira même jusqu'à confronter ses goûts à ceux de Antoinette Beaugrand mais il ne sera pas pris au sérieux tant le monde qu'il leur place devant les yeux paraît inimaginable.

Le personnage même d'Édouard sollicite le quotidien. Ce dernier ressort des limites sociales et cognitives du narrateur-personnage. En effet, le narrateur n'a pas de pouvoir cognitif sur les autres personnages, pouvoir que pourrait lui conférer la distanciation et donc le retour réflexif sur les faits vécus. Le roman nous le montre, le plus souvent, en quête de compréhension face à un monde qui lui échappe. Toute profondeur de réflexion se heurte aux conditions matérielles d'élaboration (les mots): 
J'avais la tête levée pis j'me tenais à deux mains à la balustrade. J'avais jamais vu un ciel comme celui-là. En ville, on le voit pas, le ciel. Ou par petits bouts qu'on regarde d'un œil distrait. Mais là... j'ai pas les mots pour vous décrire tout ça pis ça m'enrage! (p.69)

En outre, le quotidien prend le visage de l'apparence et du trivial par le mimétisme de classe sociale qu'adoptera Édouard. Voulant calmer son manque d'assurance en situation, Édouard modèlera son comportement sur celui de la classe sociale élevée. Cependant, il le fera en accordant une importance démesurée à l'apparence dans ses moindres détails, comme si le superficiel pouvait assurer le vernis qui lui manque. Le mimétisme de prononciation interviendra fréquemment dans le roman, accentuée la touche d'exagération souhaitable pour provoquer le rire:

Je commençais à être pas mal énervé parce que je n'arrivais pas à comprendre le tableau des départs quand mon porteur s'est arrêté net. "Vous allez à Paris?" Je lui ai répondu avec un accent qui s'approchait le plus possible du sien: "Ceurtaineument!" et je lui ai montré mon billet..."Ah! monsieur voyage en première! Monsieur est un bourgeois!" Ça faisait drôle de me faire dire ça, chez nous chus un tout nu! "Monsieur ne se mêle pas à la canaille!" Si seulement il savait que c'est ma spécialité! (p.180)

Ajoutons que la tendance d'Édouard à idéaliser le monde, surtout celui du cinéma et du théâtre à travers lequel, d'ailleurs, il s'imagine la culture française, vient en contraste avec les dures réalités de sa vie quotidienne. Cela se manifeste dans ce passage où il déplore l'ennui des fêtes à bord et leur manque d'envergure:

Dans un film (j'en reviens toujours à ça mais c'est là que j'ai le plus vu de monde riche), dans un film, on a les stars au premier plan qui parlent fort pi les figurants, derrière, qui font des mimiques pour pas trop faire de bruit... Dans les vraies croisières on a juste les figurants qui font des mimiques pis c'est ben déprimant! (TREMBLAY, 1984:124)

Mais la suite des événements nous montre qu'il n'en restera pas à ces propos passifs et mélancoliques: son sens de la fête éclatera lors d'un bal masqué où le naturel prendra le dessus sans pudeur. Un tel contraste permet à Tremblay d'exploiter tous les ressorts de l'humour tout en guidant la réflexion vers les contraintes sociales. 
Comme chez Clémence DesRochers, les enchaînements d'énoncés sont de nature à convoquer le quotidien tout en étant source d'humour. Les familiarités du personnage ressortent en conversation ou dans le récit d'événements comme autant d'échappées du naturel qui accentuent l'aspect trivial du propos. Le ton intimiste ou de connivence qu'emploie le narrateur pour commenter un événement a aussi un rôle à jouer; ces commentaires qui s'adressent à la belle-sœur — et à nous en dernière instance - surviennent dans ce contexte dialogal comme des sortes d'apartés:

- J'espère seulement que ma Lucille est assez sensible pour comprendre l'importance de tout ce qu'elle verra, même si elle ne comprend pas tout. Elle est restée dans la cabine. Elle n'est pas comme sa mère, èlle n'a pas le pied marin, la pauvre...

J'sais pas pourquoi, quand elle a dit ça, j'ai regardé ses pieds. Déformation professionnelle, probablement. Elle a pas le pied marin, elle a des pieds de marin! Sont plus grands que les miens je pense! Est enflée d'en bas comme d'en haut! (p.84)

Ce commentaire vient donc comme une confidence au lecteur et sert de transition à la suite des répliques. Le passage du figuré (sur lequel se base la locution "avoir le pied marin") au concret ("avoir de grands pieds") est un exemple de réductionnisme qu'effectue systématiquement le narrateur-personnage. Le raisonnement "terreà-terre" qui en ressort témoigne de la tendance de ce personnage d'appartenance sociale modeste et de références culturelles limitées à tout ramener à sa portée afin de rendre la situation intelligible. Les stéréotypes culturels qui se manifestent lors de la prise de contact d'Édouard, à son arrivée en France, sont dignes de mention. En décodant ce monde nouveau à travers le prisme restreint de son univers quotidien: "Mais Paris est une vieille ville alors que nous, en Amérique du Nord, on n'a pas encore eu le temps de se salir!" (p. 227).

Édouard affiche les limites désarmantes de sa compréhension en même temps que se joue, dans le roman, une affirmation basée sur une opinion commune, ou doxa, dans sa dimension répétitive et insuffisante. 
Dans cette oeuvre de Tremblay, le quotidien prend le visage du naturel, tour à tour renié puis assumé pleinement quand il quitte le rôle social contraignant qu'il s'impose en voulant masquer ses origines; le personnage se défoule alors dans un rôle-spectacle semblable à celui du travesti dans lequel il a l'habitude d'élever son quotidien et de cultiver l'illusion. Une telle rhétorique, qui scande le cliché et le place dans une ironie de situation qui s'étend à tout le roman, propulse le quotidien vers des revendications d'allure identitaire. En effet, aussi limitatives qu'apparaissent les particularités régionales, elles finissent par conquérir des droits dans ce roman; le naturel provocateur et néanmoins revendiqué, qui s'associe à l'identité sexuelle, l'origine sociale et nationale, ne va pas sans rappeler le rôle dévolu au joual, comme variété linguistique populaire, dans certains écrits littéraires québécois d'un passé récent en tant que valorisation de l'identité nationale. ${ }^{4}$

\section{Conclusion}

En conclusion, à l'opposé de l'épopée québécoise, marquante à une certaine époque de la littérature québécoise (NEPVEU, 1999:2021), une tendance moderne se profile qui réinvestit le quotidien dans le littéraire. Par des développements qui s'appesantissent sur le détail, ramènent le général au particulier, sans prétendre aux sentiments élevés, sans briguer l'originalité, une telle écriture chez les auteurs étudiés s'est donnée pour figures dominantes celles à portée textuelle, basées sur des discours d'emprunt comme le cliché et l'ironie. Elle pourrait passer pour une anti-rhétorique tant elle s'écarte des canons littéraires habituels cultivant l'esthétisme formel dans la charge des figures et la grandiloquence associée. Pourtant, il n'en résulte pas

${ }^{4}$ Nous rappelons que la pièce de théâtre, Les Belles Scuurs (1968) de Michel Tremblay était au cœur de ce débat. 
moins une structure de texte fortement organisée qui dépasse les spécificités génériques. D'autres auteurs québécois mériteraient d'être étudiés sur le plan de la rhétorique du quotidien, que cette dernière se manifeste par des moyens semblables à ceux étudiés dans le présent travail ou différemment: pensons à François Barcelo ou à Jacques Poulin, par exemple; il s'agit là d'une voie fructueuse qui reste à exploiter.

Comme nous l'avons montré, sous des abords anodins, les monologues de Clémence DesRochers condensent plusieurs procédés rhétoriques exploités systématiquement et savamment dosés. Ces procédés, bien connus par ailleurs comme l'ironie, le goût du paradoxe, le cliché, ne peuvent être abordés isolément. Ils ne font qu'un avec cet univers familier qui ne sert pas uniquement de toile de fond aux monologues, mais se construit sur le plan de la ressemblance et de la différence: conforme à nos attentes, mais conjugué avec des procédés déstabilisants que sont l'ironie, le cliché et tous les lieux de l'exagération qui s'y ajoutent. Ainsi construit, il place le lecteur-spectateur dans une position d'aller-retour entre la sympathie que l'on voue aux personnages et la distance que suscite l'humour. Des Nouvelles d'Édouard fait usage de procédés semblables tout en convoquant, cette fois, le commentaire qui souligne l'ancrage dans la réalité quotidienne du personnage.

Adoptant une vision apparemment limitative, triviale, l'écriture se fait appréhension du réel et suggère, à travers la trame narrative, une réévaluation de nos habitudes de vie, de nos réflexes discursifs, de notre comportement. La rhétorique du quotidien, par un réductionnisme exacerbé ou une transgression des normes littéraires, devient une autre façon pour l'auteur d'amener le lecteur à une distance critique et de déployer une esthétique renouvelée. 


\section{Bibliographie}

ADAMS, J.-K. Pragmatics and fiction. Amsterdam: John Benjamins, 1985.

ALLARD, Y. Introduction à une bibliographie sur l'humour, Paralittératures. Montréal: La Centrale des Bibliothèques, 1979.

AMOSSY, R., HERSCHBERG PIERROT P.. Stéréotypes et clichés. Paris: Nathan Université, 1997.

FORGET, D. Figures de pensée, figures du discours, à paraître chez Nota bene. Québec, 2000.

LARTHOMAS, P. Le langage dramatique. Paris: Presses Universitaires de France, 1972.

MAILHOT, L. Le monologue québécois, Canadian Literature, $\mathrm{n}^{\circ}$ 58, 26-38, 1973.

MAILHOT, L. et MONTPETIT, D.-M. Monologues québécois 1890-1980. Ottawa: Les Editions Leméac, 1980.

MORIN, V. L'histoire drôle, Communications, n 8, 102-120, 1966.

NEPVEU, P. L'Écologie du réel. Montréal: Boréal, 1999.

\section{Textes étudiés}

DESROCHERS, C. On a fait un ben beau voyage franch'ment hein Armand?, $L a$ Grosse Tête. Montréal: Leméac, 1973.

DESROCHERS, C. Valentine, Saint-François et la publicité, J'haï écrire! Laval: Éditions Trois, 1986.

TREMBLAY, M. Des Nouvelles d'Édouard. Montréal: Leméac, 1984. 